\title{
Injection of Gold Ions in the AGS Booster with Linear Coupling*
}

\author{
C. Gardner, L. Ahrens, T. Roser, K. Zeno, BNL, Upton, NY 11973, USA
}

\begin{abstract}
Linear Coupling, introduced by skew quadrupoles, has been used for several years to enhance the multi-turn injection efficiency of gold and other heavy ions in the AGS Booster. In this paper we describe our latest measurements of the injection process and compare with models.
\end{abstract}

\section{INTRODUCTION}

An important consideration in our effort to meet the intensity requirements for the Relativistic Heavy Ion Collider (RHIC) has been the efficiency of the multi-turn injection of heavy ions in the AGS Booster. A number of years ago we introduced linear coupling (using existing skew quadrupoles) into our injection scheme [1] and found, as others have [2], that one can significantly enhance the injection efficiency. The principle parameters of the scheme are the tunes, the skew quadrupole current, and the position and angle of the injection bump. In the past we have simply tuned these parameters on the basis of intensity using models of the injection process as a general guide. However, recently we developed a non-destructive way to observe the turn-by-turn evolution of the injected beam and this has paved the way for more detailed tuning and analysis of the injection parameters. We report here the results of our efforts and what we hope to accomplish for the upcoming commissioning of RHIC.

Heavy ion beams are injected into the Booster by means of an electrostatic inflector and a programable injection bump. The injection bump initially places the closed orbit near the septum at the exit of the inflector. As beam is injected and begins to circulate, the orbit must be moved away to keep the circulating beam from hitting the septum. The incoming beam is therefore deposited into a series of phase space layers surrounding the orbit as the bump collapses. The process continues until the orbit is so far from the septum that any additional beam injected will be outside the acceptance of the machine.

The number of turns that can be injected into a given layer will depend on the tunes and the coupling introduced by the skew quadrupoles. By judicious choice of these parameters, one can maximize the time that the circulating beam stays clear of the septum. Of course, the introduction of coupling has a price; it increases the size of the vertical envelope of the beam. This means that in the dipoles of the Booster lattice, where the vertical beta function is large and the vertical aperture is small, one can start to lose beam if too much coupling is introduced. Finding the optimum settings for the tunes and the skew quadrupole current

\footnotetext{
"Work supported by the U.S. Department of Energy.
}

therefore requires keeping track of the vertical beam envelope in the dipoles as well as the horizontal envelope at the septum.

We assume that the beam distribution emerging from the inflector is contained in a four-dimensional beam ellipsoid with an emittance small compared to the machine acceptance. At any point in the machine, the horizontal and vertical oscillation envelopes of the center of the ellipsoid are given by

$$
|X| \leq \sqrt{d^{2} \beta_{1} \epsilon_{1}}+\sqrt{\beta_{x 2} \epsilon_{2}}
$$

and

$$
\left|Y^{-}\right| \leq \sqrt{\beta_{y 1} \epsilon_{1}}+\sqrt{d^{2} \beta_{2} \epsilon_{2}}
$$

where

$$
\begin{aligned}
& \beta_{x 2}=\beta_{2} W_{22}^{2}+2 \alpha_{2} W_{22} W_{12}+\gamma_{2} W_{12}^{2} \\
& \beta_{y 1}=\beta_{1} W_{11}^{2}-2 \alpha_{1} W_{11} W_{12}+\gamma_{1} W_{12}^{2}
\end{aligned}
$$

The parameters $\alpha_{1}, \beta_{1}, \gamma_{1}, \alpha_{2}, \beta_{2}, \gamma_{2}, W_{11}, W_{12}, W_{21}$, $W_{22}$, and $d$ are determined by the one-turn transfer matrix at the given point and can be obtained from the MAD code [3]. $\epsilon_{1}$ and $\epsilon_{2}$ are the Courant-Snyder Invariants associated with the two normal-modes of oscillation of the beam ellipsoid center about the closed orbit.

The extent of the beam ellipsoid with respect to its center is defined by a four-by-four symmetric positive-definite matrix, $\mathbf{E}$, which we assume is matched to the lattice. The maximum possible horizontal and vertical excursions of a beam particle with respect to the center of the ellipsoid are then given by

$$
|U-X| \leq \sqrt{\epsilon_{b} E_{11}}, \quad|V-Y| \leq \sqrt{\epsilon_{b} E_{33}}
$$

where $E_{11}$ and $E_{33}$ are matrix elements of $\mathbf{E}$ and $\epsilon_{b}$ is the beam ellipsoid emittance. (Here $U$ and $V$ are the horizontal and vertical coordinates of a beam particle with respect to the closed orbit.) The beam envelope at a given point in the ring is then defined by the equations

$$
|U| \leq \sqrt{d^{2} \beta_{1} \epsilon_{1}}+\sqrt{\beta_{x 2} \epsilon_{2}}+\sqrt{\epsilon_{b} E_{11}}
$$

and

$$
|V| \leq \sqrt{\beta_{y 1} \epsilon_{1}}+\sqrt{d^{2} \beta_{2} \epsilon_{2}}+\sqrt{\epsilon_{b} E_{33}} .
$$

\section{TURN-BY-TURN MEASUREMENTS}

The horizontal and vertical positions (with respect to the closed orbit) of the beam ellipsoid center at a given point on the $n$th turn around the machine are given by

$$
X_{n}=A_{1} \cos \left(n \psi_{1}+\phi_{1}\right)+A_{2} \cos \left(n \psi_{2}+\phi_{2}\right)
$$


and

$$
Y_{n}=B_{1} \cos \left(n \psi_{1}+\eta_{1}\right)+B_{2} \cos \left(n \psi_{2}+\eta_{2}\right)
$$

where $\psi_{1}=2 \pi Q_{1}$ and $\psi_{2}=2 \pi Q_{2}$. Here we see that the motion in each plane is the superposition of two modes of oscillation, and the tunes $\left(Q_{1}, Q_{2}\right)$, amplitudes $\left(A_{1}, A_{2}\right.$, $\left.B_{1}, B_{2}\right)$ and phases $\left(\phi_{1}, \phi_{2}, \eta_{1}, \eta_{2}\right)$ are the 10 parameters that characterize the oscillations. These parameters are not all independent; the amplitudes and phases are each functions of only 4 independent parameters, the initial positions and angles $X_{0}, X_{0}^{\prime}, Y_{0}, Y_{0}^{\prime}$ of the beam ellipsoid center. Including the tunes, there are therefore only 6 independent parameters and these ultimately are what one wants to obtain from turn-by-turn measurements.

To observe the turn-by-turn evolution of the beam position, it is necessary to chop the beam so that a short pulse corresponding to each turn can be seen on PUE's (PickUp Electrodes) in the Booster ring. The chopper is located in the transport line upstream of the Booster, and consists of two parallel plates with one plate above and the other below the midplane of the beamline. The upper and lower plates are connected respectively to pulsed and DC high-voltage power supplies, and the beam is deflected vertically by applying voltages to the plates. In the past, the chopper has been set up so that only a half-turn pulse of beam is transmitted down the beamline and injected into the Booster; the turn-by-turn evolution of the half-turn can then be observed on the PUE's in the ring. In this mode of operation, beam is transmitted down the line only when the upper plate is pulsed with a voltage which just cancels a DC bias voltage applied to the lower plate. Putting only a half-turn pulse (some $7.5 \mu \mathrm{s}$ ) down the line is, of course, destructive to the Physics programs; they require the full long pulse from the ion source (typically $500-1000 \mu \mathrm{s}$ ) which corresponds to several tens of turns around the ring. However, the chopper also can be set up so that all of the long pulse is transmitted except for a half-turn portion which may be selected from any part of the long pulse. In this mode, no bias voltage is applied to the lower plate and beam is transmitted down the line except when a deflecting pulse is applied to the upper plate. The deflecting pulse produces a half-turn gap or "hole" in the long pulse that is essentially invisible to the Physics programs and at the same time provides a turn-by-turn signal on the PUE's. We have found that the turn-by-turn evolution of this "hole" is essentially the same as that of a half-turn pulse of beam by itself. This mode of operation allowed us to make several turn-by-turn measurements of the injected beam without interrupting the Physics program.

A computer program called "PIP" [4] collects and analyzes oscilliscope traces of the turn-by-turn signals obtained from a given PUE in the Booster ring. The program first obtains the position of the injected beam versus turn at the PUE; a Discrete Fourier Transform of the data then gives the normal-mode tunes and initial phases associated with the coupled betatron oscillations. Using these as starting values, the horizontal or vertical function
$(8-9)$ is fitted to the data. From the fitted parameters and the lattice parameters at the PUE and at the inflector exit, the program then calculates the initial positions and angles of the beam at injection. Figure 1 shows typical positionversus-turn data along with the fitted curve; the FFT of the data is shown in Figure 2. Here the normal-mode tunes are $Q_{1}=4.699(1), Q_{2}=4.801(1)$. With these tunes the injected beam returns to its initial position evcry 10 turns; hence, a maximum of 10 turns can be injected into a phasespace layer before the bumped orbit must be moved away from the septum. Figures 3 and 4 are the corresponding figures for another set of data. Here the normal-mode tunes are $Q_{1}=4.736(1), Q_{2}=4.803(1)$ and the injected beam returns to its initial position every 15 turns.

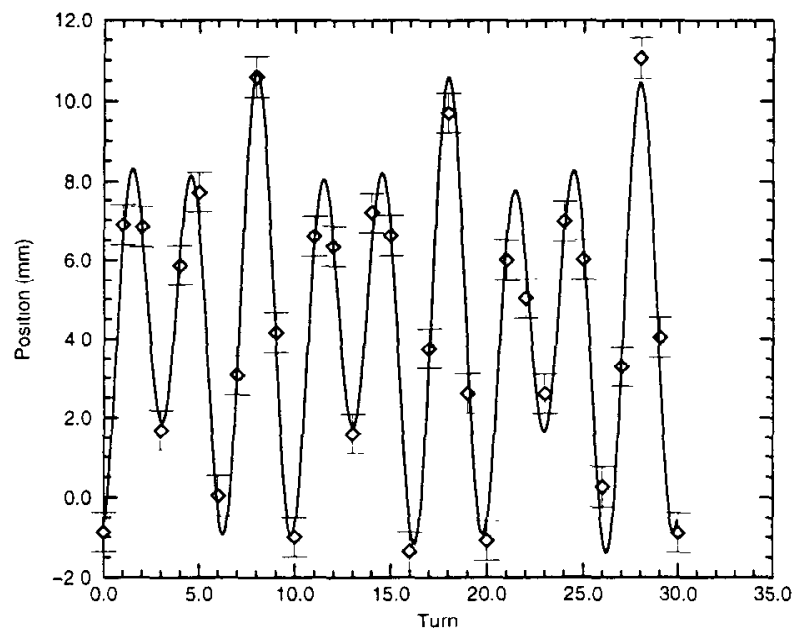

Figure 1: Position-vs-Turn Data and Fitted Curve.

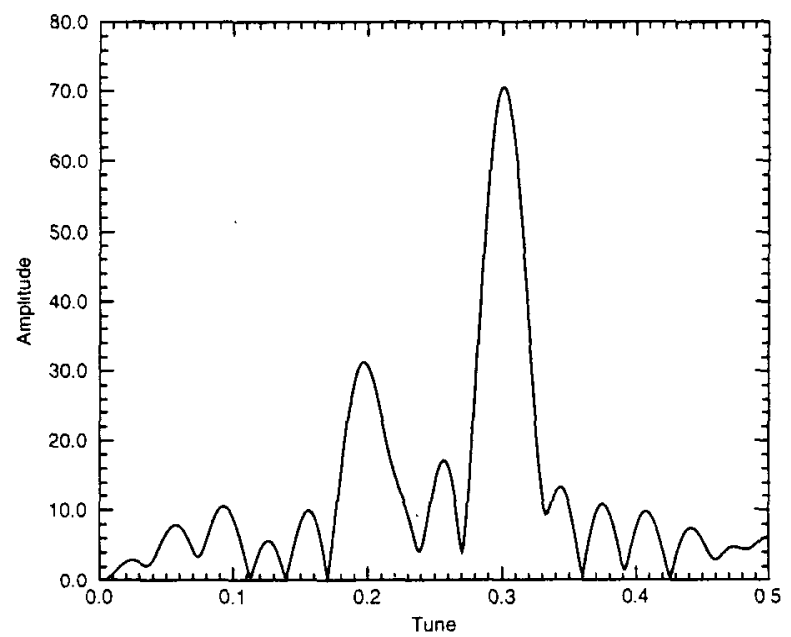

Figure 2: FFT of Position-vs-Turn Data.

\section{INJECTION SETUP}

The turn-by-turn measurements provide a useful tool for setting up various coupled injection schemes. Although 


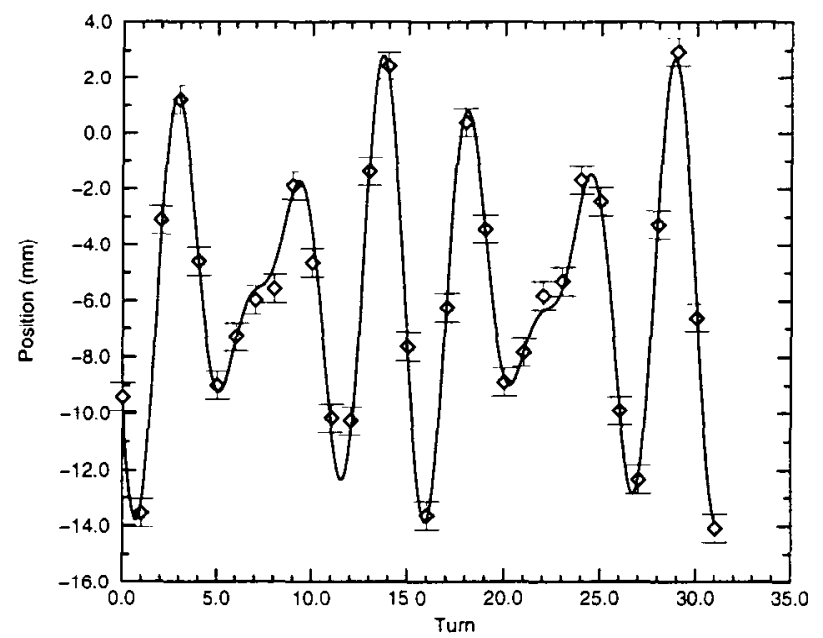

Figure 3: Position-vs-Turn Data and Fitted Curve.

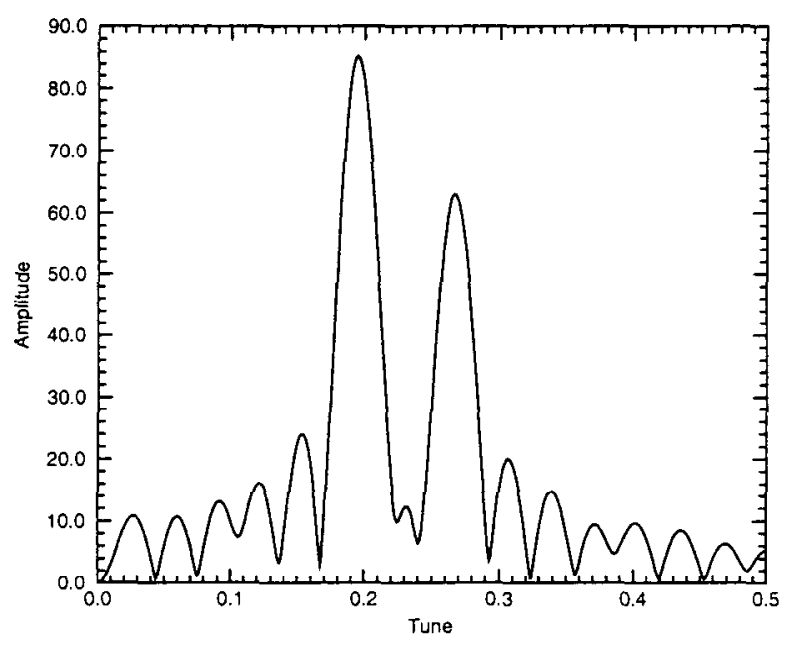

Figure 4: FFT of Position-vs-Turn Data.

not yet implemented in practice, one can proceed in the following way. A given scheme is defined by the settings of the unperturbed (i.e. uncoupled) horizontal and vertical tunes, $Q_{x}$ and $Q_{y}$, and the skew quadrupole current, $I$. These determine the normal-mode tunes, $Q_{1}$ and $Q_{2}$, and the amount of coupling between the two planes. Using the MAD code to obtain the necessary parameters, equations (6-7) then give the maximum horizontal and vertical extent of the beam at the septum and at the vertical beta maximums in the Booster. This allows one to determine how far the orbit has to be moved to keep the beam from hitting the septum. It also tells us when the vertical envelope becomes too large in the Booster dipoles. We define $X_{\mathrm{M}}$ to be the maximum horizontal excursion of the beam ellipsoid center on its passes by the septum at the inflector exit; $X_{\mathrm{M}}+\sqrt{\epsilon_{b} E_{11}}$ must not exceed $45 \mathrm{~mm}$ in order for the injected beam to be inside the Booster acceptance once the injection bump has collapsed completely. Similarly, we define $Y_{M}$ to be the maximum vertical excursion of the ellipsoid center at the vertical beta maxi- mums in the Booster; $Y_{M}+\sqrt{\epsilon_{b} E_{33}}$ must not exceed 33 $\mathrm{mm}$. (We assume that the closed orbit is centered in the dipoles.) Assuming the incoming beam has an emittance of $1 \pi \mathrm{mm}$ milliradian in both planes, one finds that for a 10-turn scheme similar to that of Figure 1, three layers of 10 turns can be injected before the vertical beam envelope becomes too large in the Booster dipoles. For a 15-turn scheme similar to that of Figure 3, one can inject two layers of 15 turns before the vertical envelope becomes too large. To put beam into additional layers, one must reduce the coupling so that the vertical envelope does not increase any further. One way to do this is to move the uncoupled tunes further apart. (In practice one can change the tunes faster than the skew quadrupole current.) For the 15-turn scheme, a shift of the vertical tune reduces the coupling and allows two more layers to be injected with 9 and 19 turns respectively. In principle, a total of 62 turns can then be injected; the relevant parameters are summarized in Table 1. Here the normal-mode tunes are $Q_{1}=4+11 / 15=4.733, Q_{2}=4+12 / 15=4.800$, and the required skew quadrupole current is 3.7 Amps. $X_{b}$ and $X_{\mathrm{b}}^{\prime}$ are the horizontal position and angle of the bumped orbit at the inflector exit; the units are $\mathrm{mm}$ and milliradians. In practice we have not yet injected this many turns. Anec-

Table 1: Coupled Injection Scheme

\begin{tabular}{|c|c|c|c|c|c|}
\hline \multicolumn{6}{|c|}{$Q_{x}=4.7767, Q_{y}=4.7567$} \\
\hline Layer & $X_{\mathrm{b}}$ & $X_{\mathrm{b}}^{\prime}$ & Turns & $X_{\mathrm{M}}$ & $Y_{\mathrm{MI}}$ \\
\hline 1 & 41.81 & 6.58 & 4 & 10.00 & 10.32 \\
2 & 34.19 & 5.38 & 15 & 17.61 & 18.19 \\
3 & 26.58 & 4.18 & 15 & 25.23 & 26.05 \\
\hline \multicolumn{6}{|c|}{$Q_{x}=4.7767, Q_{y}=4.7000$} \\
\hline 4 & 18.96 & 2.97 & 9 & 32.85 & 22.89 \\
5 & 11.34 & 1.78 & 19 & 40.47 & 28.20 \\
\hline
\end{tabular}

dotal evidence suggests that the stripping foil in the beamline upstream of the Booster may significantly increase the beam emittance, so that the number assumed above may be too small. Nevertheless, we believe that our measurement technique and an injection scheme similar to the one above will prove useful during the upcoming commissioning of RHIC.

\section{REFERENCES}

[1] T. Roser, "Multi-turn Injection with Coupling", AGS/AD/Tech. Note No. 354, November 7. 1991.

[2] K. Schindl and P. Van der Stok, IEEE Transactions on Nuclear Science, Vol. NS-24, No. 3, June 1977, pp. 1390-1392.

[3] H. Grote and F. C. Iselin. "The MAD Program Version 8.4 User's Reference Manual", CERN/SL/90-13 (AP), 27 August 1991.

[4] C. J. Gardner, "Turn-by-Turn Analysis of Proton and Gold Beams at Injection in the AGS Booster", Proceedings of the 1999 Particle Accelerator Conference. 